\section{L'Actualité économique}

L'ACTUALITÉ

ÉCONOMIQUE

\section{Introduction du numéro spécial sur le thème de l'organisation industrielle}

\section{Abraham Hollander}

Volume 65, numéro 1, mars 1989

Organisation industrielle

URI : https://id.erudit.org/iderudit/601476ar

DOI : https://doi.org/10.7202/601476ar

Aller au sommaire du numéro

Éditeur(s)

HEC Montréal

ISSN

0001-771X (imprimé)

1710-3991 (numérique)

Découvrir la revue

Citer ce document

Hollander, A. (1989). Introduction du numéro spécial sur le thème de

l'organisation industrielle. L'Actualité économique, 65(1), 5-7.

https://doi.org/10.7202/601476ar d'utilisation que vous pouvez consulter en ligne. 


\title{
Introduction du numéro spécial sur le thème de l'organisation industrielle
}

\author{
Abraham HOLLANDER \\ Université de Montréal*
}

Les essais que nous publions dans ce numéro spécial abordent divers thèmes de recherche en organisation industrielle. La majorité des contributions traite de questions d'économie appliquée, surtout de politique industrielle et de concurrence. Cependant, des problèmes de nature plus théorique, comme la détermination des choix des variables de décision en équilibre d'oligopole, ne sont pas négligés.

Dans un premier article Jacquemin situe la nouvelle économie industrielle par rapport à l'économie industrielle traditionnelle. L'auteur caractérise la première par une utilisation croissante des outils de micro-économie et de la théorie des jeux. Il attribue la multiplication des modèles au souci de préserver la richesse des informations apportées par les études de cas, qui depuis longtemps font partie du bagage analytique des économistes industriels. Un certain éclectisme est le résultat inévitable d'une volonté de «coller» à la réalité. Une deuxième caractéristique que l'auteur attribue à la nouvelle économie industrielle est l'importance qui est donnée aux activités stratégiques par lesquelles les entreprises façonnent leur environnement. Il montre comment l'économie industrielle aborde la question de savoir si les formes de marchés et les structures des entreprises constituent une adaptation efficace à l'environnement ou sont plutôt le résultat de stratégies qui visent à modifier les règles du jeu concurrentiel à l'avantage de firmes puissantes. Il pose ensuite la question difficile de savoir à quels critères devrait obéir une politique industrielle optimale. Sans prendre position il trace de façon très nette le cercle à l'intérieur duquel doit être tenu le débat sur cette question.

Comme le précisent Encaoua et Santini les interventions publiques destinées à corriger des défaillances de marché prennent trois formes, la fiscalité, la réglementation et la nationalisation. Les auteurs passent en revue les défaillances du marché et les défaillances de l'intervention publique qui sont à la source du choix du mode de propriété. Ils traitent de façon détaillée des modalités de mise en oeuvre des privatisations en France, et en font un bilan intérimaire pour finalement conclure que si les privatisations sont une condition nécessaire de libéralisme économique elles ne constituent pas en même temps une condition suffisante.

*Département de sciences économiques. 
La question de savoir comment les gouvernements interviennent dans les mécanismes de marché intéresse également Dalpé et DeBresson. Ces auteurs explorent le rôle du secteur public canadien à la fois comme acheteur et comme utilisateur de nouvelles technologies. Ils constatent que la quote-part des ventes de produits destinées au secteur public est beaucoup plus importante pour les industries intensives en recherche que pour l'ensemble des industries. Qui plus est, le secteur public occupe une place encore plus prépondérante dans la genèse de premières mondiales. Ils en concluent que le secteur public oriente le développement technologique et ils proposent un nombre de questions qui devraient servir à clarifier ce rôle.

Une étude d'une intervention publique fédérale sous forme de réglementation d'un secteur nationalisé provincial nous est présentée par Jean-Thomas Bernard. L'auteur explore dans le cadre d'un modèle formel l'impact d'une disposition de la Loi sur l'Office national de l'énergie qui précise qu'un producteur canadien d'électricité ne peut exporter à un prix inférieur au prix exigé des Canadiens pour un service équivalent dans des régions connexes. Son analyse porte sur l'efficacité économique de cette disposition. Il démontre qu'en présence de vendeurs provinciaux disposant d'un pouvoir de marché, la disposition augmente le bien-être au Canada et cela dans des conditions très plausibles.

La difficulté de déterminer un degré optimal d'intervention est également étudiée par Ponssard et Tanguy. Ils constatent que d'une part, la complexité des problèmes empêche une planification totale avec instructions contingentées alors que d'autre part, un mécanisme décentralisé requiert le consensus à propos des règles du jeu ainsi qu'un savoir commun qui est loin d'être parfait. Les auteurs cherchent à apporter des éléments de réponse à la question de savoir si on peut «formuler des règles qui ne soient pas totalement déterministes, en laissant subsister des possibilités d'ajustement, et telles qu'elles aient un rôle positif face à un problème local... mais dans le cadre d'une perception limitée des enjeux globaux. » Les réflexions des auteurs aboutissent à une conception interactive de la rationalité qui requiert l'abandon de toute prétention d'obtenir des lois générales. Le rôle de l'économiste doit dès lors se limiter à contribuer au savoir commun «en étant sûr qu'on n'a pas l'ensemble des éléments et en invitant constamment les hommes d'entreprise à la vigilance par rapport aux recommandations que l'on fait. »

Un domaine important de l'organisation industrielle est l'analyse des équilibres d'oligopole. On affirme souvent que ces équilibres sont indéterminés, et ce pour deux raisons. Premièrement, ils dépendent de façon critique de rôles de suiveur ou de leader que l'on attribue aux firmes. Or, si on fait exception des modèles d'entrée où les coûts irrécupérables jouent un rôle important, on est obligé de constater que l'assignation des rôles demeure exogène aux modèles et repose sur des fondements théoriques peu solides.

Une deuxième faiblesse des modèles d'oligopole est celui du choix de la variable de décision. Dans le modèle traditionnel les firmes choisissent soit, une 
variable prix soit, une variable quantité. L'autre variable s'ajuste toujours de manière à ce que la combinaison des deux se trouve sur la fonction de demande. Or, l'observation des pratiques commerciales des entreprises suggère qu'il serait opportun de construire des modèles où les firmes choisissent à la fois le prix et la quantité. L'article de Haurie s'adresse à la première de ces difficultés que nous posent les modèles d'oligopole en endogénisant le choix des rôles. Il développe un modèle dans lequel un duopoleur peut temporairement jouer un rôle de leader au sens de Stackelberg s'il réussit à faire une percée technologique avant son rival. Il continuera d'assumer ce rôle tant et aussi longtemps qu'il possède un avantage technologique. Le moment précis où est réalisée l'innovation technologique est gouvernée par une loi de probabilité qui est influencée par un niveau d'investissement en R\&D. Son modèle conserve la propriété de symétrie, car a priori les deux firmes ont les mêmes chances de devenir leader.

Boyer et Moreaux s'intéressent à l'existence d'un équilibre de duopole de type meneur-suiveur où les variables de choix sont à la fois les prix et les quantités. Ils considèrent un modèle linéaire de biens différenciés et caractérisent la demande résiduelle de chaque firme dans le contexte de cette stratégie à deux variables. Ils illustrent également l'existence de rationnement à l'équilibre.

Les deux derniers articles de ce numéro traitent de politiques de concurrence au Canada. L'ancienne Loi relative aux enquêtes sur les coalitions a été amendée et est devenue en 1986 la Loi sur la concurrence. Les amendements traduisent un nouvel environnement caractérisé par une internationalisation plus forte de la concurrence. Ils traduisent également un nombre de résultats théoriques récents qui mettent l'accent sur le comportement des entreprises plutôt que sur la structure des marchés. Les Brenner insistent sur le fait que les indices conventionnels de concurrence, provenant des modèles néoclassiques, favorisent une optique d'équilibre de long terme et sont insuffisants pour décrire la rivalité telle quelle est vécue dans les marchés. En effet, celle-ci s'exerce dans un monde où l'esprit d'entreprise et l'innovation, c'est-à-dire des éléments de déséquilibre, jouent le rôle prépondérant. Dans un premier temps les auteurs situent l'innovation dans les processus de concurrence et explorent les difficultés auxquelles on s'expose en voulant juger un impact concurréntiel à partir des indicateurs conventionnels. Dans un deuxième temps, ils se servent d'exemples de fusion et de compression de marges bénéficiaires qui ont donné lieu à des poursuites judiciaires pour appuyer une conclusion voulant qu'en ignorant l'innovation les tribunaux peuvent manquer de considérer un aspect parmi les plus importants de la rivalité entre les firmes.

Hollander se penche sur les conflits entre la Loi sur la concurrence et la Loi sur les mesures spéciales d'importation, en particulier le traitement différent qu'elles accordent à la discrimination dans les marchés internes et externes. Il montre, en invoquant un nombre de causes récentes, qu'une perception très répandue voulant que l'anti-dumping est une application au marché international d'interdictions de discriminer sur le marché national, que l'on retrouve déjà dans les lois sur la concurrence, est inexacte. 